Commun. Korean Math. Soc. 26 (2011), No. 3, pp. 463-472

DOI 10.4134/CKMS.2011.26.3.463

\title{
EVOLUTION EQUATIONS ASSOCIATED WITH TIME-DEPENDENT SUBDIFFERENTIALS
}

\author{
KIYEOn SHIn AND JingYo JEONG
}

\begin{abstract}
In this paper we study the solvability of parabolic equations governed by the difference of time dependent subdifferential and time independent subdifferential in reflexive Banach spaces.
\end{abstract}

\section{Introduction}

Let $X$ and $X^{*}$ be a real Banach space and its dual space, respectively, and let $H$ be a Hilbert space whose dual space $H^{*}$ is identified with itself such that $X \hookrightarrow H \equiv H^{*} \hookrightarrow X^{*}$ with continuous and densely defined canonical injections.

The main purpose of this paper is to study the solvability of the following abstract Cauchy problem in $X-X^{*}$ setting:

$(\mathrm{CP})\left\{\begin{array}{l}\frac{d u}{d t}(t)+\partial \varphi(t, u(t))-\partial \psi(u(t)) \ni f(t) \quad \text { in } X^{*}, 0<t<T \\ u(0)=u_{0},\end{array}\right.$

where $\partial \varphi(t, \cdot), \partial \psi: X \rightarrow 2^{X^{*}}$ are the subdifferentials of a time-dependent lower semicontinous convex function $\varphi(t, \cdot): X \rightarrow(-\infty, \infty]$ with $\varphi(t, \cdot) \not \equiv+\infty$ for $t \in(0, T)$ and of a lower semicontinous convex function $\psi: X \rightarrow(-\infty, \infty]$ with $\psi \neq \equiv+\infty$, respectively. And $f:(0, T) \rightarrow X^{*}$ is given.

(CP) is studied by Koi-Watanabe [9], Ishii [7] and Ôtani [11] in the Hilbert space framework for the existence and the asymptotic behavior of strong solutions. Recently, Akagi and Ôtani [3] has considered the autonomous problem under the subcritical growth condition to get a local weak solution in reflexive Banach space.

For the case of time dependent $\varphi(t, \cdot)$, Ôtani [11], Kenmochi [8] proved the existence of a strong solutions on $[0, T]$ of the Cauchy problem for the equation $d u(t) / d t+\partial \varphi(t, u(t))+B(t, u(t)) \ni f(t)$ in Hilbert setting, i.e., $X=X^{*}=H$ by applying nonlinear interpolation theory.

Received May 19, 2009.

2010 Mathematics Subject Classification. 34G25, 35K55, 35K65.

Key words and phrases. Cauchy problem, time dependent subdifferential, reflexive Banach space.

This work was supported for two years by Pusan National University Research Grant.

(C)2011 The Korean Mathematical Society 
Recently, Akagi and Ôtani [3] considered the existence of strong solution of Cauchy problem for $d u(t) / d t+\partial \varphi^{1}(u(t))-\partial \varphi^{2}(u(t)) \ni f(t)$ in $X-X^{*}$ setting which should remedy the deficiency in Hilbert space setting for applying our abstract results to the PDE problems. With their results, we prove the existence of strong solutions of (CP) on $[0, T]$ by imposing a $t$-smoothness condition on $\varphi(t, \cdot)$ in addition to the similar assumptions as in [3]. The method of our proof relies on some approximations of $(\mathrm{CP})$ to obtain the approximate solutions which converges.

This paper is composed of three sections. In the next section, we summarized the relevant material on subdifferential operators. Section 3 is devoted to state our main results on the existence of strong solutions for (CP).

\section{Preliminaries}

Let $X$ be a real reflexive Banach space and let $X^{*}$ be its dual. We assume that there exists a real Hilbert space $H$ whose dual space $H^{*}$ is identified with $H$ such that $X \subset H \equiv H^{*} \subset X^{*}$, where the natural injection from $X$ into $H$ as well as that from $H^{*}$ into $X^{*}$ are densely defined and continuous.

To formulate our results, we need the notion of subdifferential operators from a Banach space $X$ into its dual $X^{*}$ defined below.

Let $\Phi(X)$ be the set of all proper lower semicontinuous convex functions $\varphi$ from $X$ into $(-\infty,+\infty]$, where "proper" means that the effective domain $D(\varphi)$ of $\varphi$ defined by $D(\varphi)=\{u \in X: \varphi(u)<+\infty\}$ is not empty. The subdifferential $\partial_{X} \varphi(u)$ of $\varphi$ at $u$ in $X$ is defined by

$$
\partial_{X} \varphi(u)=\left\{f \in X^{*}: \varphi(v)-\varphi(u) \geq_{X^{*}}\langle f, v-u\rangle_{X} \text { for all } v \in D(\varphi)\right\}
$$

with domain $D\left(\partial_{X} \varphi\right)=\left\{u \in D(\varphi): \partial_{X} \varphi(u) \neq 0\right\}$, where $X^{*}\langle\cdot, \cdot\rangle_{X}$ denote the duality pairing between $X$ and $X^{*}$. For simplicity of notation, we write $\partial \varphi$ and $\langle\cdot, \cdot\rangle$ instead of $\partial_{X} \varphi$ and $X^{*}\langle\cdot, \cdot\rangle_{X}$, respectively, if no confusion arises. It is well known that the graph of every subdifferential operator $\partial \varphi$ becomes maximal monotone in $X \times X^{*}$ (see Barbu [5] for more details of maximal monotone operators).

In particular, if $X$ is a Hilbert space $H$ and $\varphi \in \Phi(H)$, then

$$
\partial_{H} \varphi(u)=\left\{f \in H: \varphi(v)-\varphi(u) \geq(f, v-u)_{H} \text { for all } v \in D(\varphi)\right\},
$$

where $(\cdot, \cdot)_{H}$ denotes the inner product of $H$. Furthermore, the Moreau-Yosida regularization $\varphi_{\lambda}$ of $\varphi$ is defined as follows:

$$
\varphi_{\lambda}(u):=\inf _{v \in H}\left\{\frac{1}{2 \lambda}|u-v|_{H}^{2}+\varphi(u)\right\}, \quad \forall u \in H, \forall \lambda>0 .
$$

The following proposition provides some useful properties of Moreau-Yosida regularizations. 
Proposition 2.1. Let $\varphi \in \Phi(H)$. Then $\varphi_{\lambda}$ becomes a Fréchet differentiable convex function from $H$ into $\mathbb{R}$ and is characterized by

$$
\varphi_{\lambda}(u)=\frac{1}{2 \lambda}\left|u-J_{\lambda} u\right|_{H}^{2}+\varphi\left(J_{\lambda} u\right)=\frac{\lambda}{2}\left|\left(\partial_{H} \varphi\right)_{\lambda}(u)\right|_{H}^{2}+\varphi\left(J_{\lambda} u\right),
$$

where $\left(\partial_{H} \varphi\right)_{\lambda}$ and $J_{\lambda}$ are the Yosida approximation and the resolvent of $\partial_{H} \varphi$, respectively, i.e., $J_{\lambda}=\left(I+\lambda \partial_{H} \varphi\right)^{-1}$ and $\left(\partial_{H} \varphi\right)_{\lambda}=\left(I-J_{\lambda}\right) / \lambda$. Moreover, $\partial_{H}\left(\varphi_{\lambda}\right)=\left(\partial_{H} \varphi\right)_{\lambda}$, where $\partial_{H}\left(\varphi_{\lambda}\right)$ denotes the subdifferential (Fréchet derivative) of $\varphi_{\lambda}, \varphi\left(J_{\lambda} u\right) \leq \varphi_{\lambda}(u) \leq \varphi(u)$ for all $u \in H, \lambda>0$ and $\varphi_{\lambda}(u) \rightarrow$ $\varphi(u)$ as $\lambda \rightarrow 0$ for all $u \in H$.

Proposition 2.2. Let $\varphi \in \Phi(H)$ and suppose that $u \in W^{1,2}(0, T ; H), u(t) \in$ $D\left(\partial_{H} \varphi\right)$ for a.e. $t \in(0, T)$ and that there exists $h \in L^{2}(0, T ; H)$ such that $h(t) \in \partial_{H} \varphi(u(t))$ for a.e. $t \in(0, T)$. Then the function $t \mapsto \varphi(u(t))$ is absolutely continuous on $[0, T]$ and the following holds;

$$
\frac{d}{d t} \varphi(u(t))=\left(h(t), \frac{d u(t)}{d t}\right)_{H}, \forall h(t) \in \partial_{H} \varphi(u(t)) \text { for a.e. } t \in(0, T) .
$$

\section{Main result}

In the paper, we are concerned with strong solution of $(\mathrm{CP})$ in the following sense.

Definition. A function $u \in C\left([0, T] ; X^{*}\right)$ is said to be a strong solution of $(\mathrm{CP})$ on $[0, T]$, if the following conditions are satisfied;

(1) $u(t)$ is a $X^{*}$-valued absolutely continuous function on $[0, T]$.

(2) $u(t) \rightarrow u_{0}$ strongly in $H$ as $t \rightarrow 0+$.

(3) $u(t) \in D(\partial \varphi(t, \cdot)) \cap D(\partial \psi)$ for a.e. $t \in(0, T)$. And there exist $g(t) \in$ $\partial \psi(u(t))$ and $h(t) \in \partial \varphi(t, u(t))$ satisfying;

$$
\frac{d u(t)}{d t}+h(t)-g(t)=f(t), \quad \text { in } X^{*} \text { for a.e. } t \in(0, T) .
$$

Prior to present our main result, we give the following assumptions for $p \in$ $(1,+\infty)$.

(A.1) There exist functions $a, b \in W^{1, \infty}(0, T)$ and a constant $\delta>0$ such that for every $t_{0} \in[0, T]$ and $z_{0} \in D\left(\varphi\left(t_{0}, \cdot\right)\right)$, we can take a function

$$
z: I_{\delta}\left(t_{0}\right):=\left[t_{0}-\delta, t_{0}+\delta\right] \cap[0, T] \rightarrow X
$$

satisfying

(1) $\left|z(t)-z_{0}\right|_{X} \leq\left|a(t)-a\left(t_{0}\right)\right|\left(\varphi\left(t_{0}, z_{0}\right)+1\right)^{1 / p}$,

(2) $\varphi(t, z(t)) \leq \varphi\left(t_{0}, z_{0}\right)+\left|b(t)-b\left(t_{0}\right)\right|\left(\varphi\left(t_{0}, z_{0}\right)+1\right) \quad \forall t \in I_{\delta}\left(t_{0}\right)$.

(A.2) There exists a constant $C_{1}$ such that $|u|_{X}^{p} \leq C_{1}\left(\varphi_{\lambda}(t, u)+|u|_{H}^{2}\right)$ for all $u \in D(\varphi(t, \cdot)), t \in[0, T]$ and for sufficiently small $\lambda>0$.

(A.3) There exists a constant $C_{2}$ such that $|\zeta|_{X^{*}}^{p^{\prime}} \leq C_{2}\left(\varphi_{\lambda}(t, u)+1\right)$ for all $\zeta \in \partial \varphi(t, u), t \in[0, T]$ and for sufficiently small $\lambda>0$, where $p^{\prime}$ is the conjugate dual of $p$. 
(A.4) $D(\psi) \subset D\left(\partial \varphi(t, \cdot)\right.$ for all $t \in[0, T]$ and there exists a constant $C_{3}$ such that $\psi(u) \leq C_{3}\left(\varphi_{\lambda}(t, u)+1\right)$ for sufficiently small $\lambda>0$, where $\varphi_{\lambda}(t, u)$ is the Yosida approximation of $\varphi(t, u)$.

Now, our main results are stated as follows.

Theorem 3.1. Assume that (A.1)-(A.4) hold. Then for all $u_{0} \in D(\varphi(0, \cdot)$ and $f \in W^{1, p^{\prime}}\left(0, T ; X^{*}\right) \cap L^{2}(0, T ; H)$, (CP) has a strong solution $u$ on $[0, T]$ satisfying:

$$
\left\{\begin{array}{l}
u \in C_{w}([0, T] ; X) \\
u(t) \in D(\partial \varphi(t, \cdot)) \cap D(\partial \psi) \text { for a.e. } t \in(0, T), \\
h, g \in L^{p^{\prime}}\left(0, T ; X^{*}\right)
\end{array}\right.
$$

where $h, g$ are the sections of $\partial \varphi(t, \cdot), \partial \psi$, respectively. And $C_{w}([0, T] ; X)$ denotes the set of all $X$-valued weakly continuous functions on $[0, T]$.

For the proof of Theorem 3.1, we first of all introduce suitable approximation problems for (CP) in the Hilbert space $H$. To this end, we define the extension $\tilde{\varphi}(t, \cdot)$ of $\varphi(t, \cdot)$ on $H$ by

$$
\tilde{\varphi}(t, u)= \begin{cases}\varphi(t, u), & \text { if } u \in X, \\ +\infty, & \text { if } u \in H \backslash X .\end{cases}
$$

Then, by (A.2), we can easily show that $\tilde{\varphi}(t, \cdot) \in \Phi(H)$.

Now, we consider our approximation problems for $(\mathrm{CP})$ as follows;

$$
(\mathrm{CP})_{\lambda}\left\{\begin{array}{l}
\frac{d u_{\lambda}}{d t}(t)+\partial_{H} \tilde{\varphi}_{\lambda}\left(t, u_{\lambda}(t)\right)-\partial_{H} \tilde{\psi}\left(u_{\lambda}(t)\right) \ni f_{\lambda}(t) \\
\quad \text { in } H, 0<t<T \\
u_{\lambda}(0)=u_{0},
\end{array}\right.
$$

where $f_{\lambda}$ belongs to $C^{1}([0, T] ; H)$ such that $f_{\lambda} \rightarrow f$ strongly in $W^{1, p^{\prime}}\left(0, T ; X^{*}\right)$ as $\lambda \rightarrow 0+$. Also, $\tilde{\psi}$ is the extension of $\psi$ on $H$ and $\partial_{H} \tilde{\varphi}_{\lambda}(t, \cdot)$ denotes the Yosida approximation of $\partial_{H} \tilde{\varphi}(t, \cdot)$.

In considering $(\mathrm{CP})_{\lambda}$, it is essential to find some properties of $\partial_{H} \tilde{\varphi}_{\lambda}$. We prepare the next lemma for this.

Lemma $3.2([2])$. Let $\varphi(t, \cdot) \in \Phi(X)$ for $t \in[0, T]$ and $u \in X$. Suppose (A.1) holds. Then, for $t, s \in[0, T]$ with $|t-s|<\delta$, where $\delta$ is given (A.1),

$$
\begin{aligned}
& \left|J_{\lambda}(t, u)-J_{\lambda}(s, u)\right|_{H}^{2} \\
\leq & 2|a(t)-a(s)|\left(\left|u-J_{\lambda}(t, u)\right|_{X^{*}}\left\{\varphi\left(s, J_{\lambda}(s, u)\right)+1\right\}^{1 / p}\right. \\
& \left.+\left|u-J_{\lambda}(s, u)\right|_{X^{*}}\left\{\varphi\left(t, J_{\lambda}(t, u)\right)+1\right\}^{1 / p}\right) \\
& +2 \lambda|b(t)-b(s)|\left\{\varphi\left(t, J_{\lambda}(t, u)\right)+\varphi\left(s, J_{\lambda}(s, u)\right)+2\right\},
\end{aligned}
$$

where $J_{\lambda}(t, \cdot)$ denotes the resolvent of $\partial_{H} \tilde{\varphi}(t, \cdot)$. 
Since $\partial_{H} \tilde{\varphi}_{\lambda}(t, \cdot)$ is Lipschitz continuous on $H$ and $\partial_{H} \tilde{\varphi}_{\lambda}(\cdot, u)$ is continuous on $[0, T]$ by the above lemma, it is well known (see Brézis [6], Theorem 1.4) that there exists a unique strong solution $u_{\lambda}$ on $(\mathrm{CP})_{\lambda}$ on $[0, T]$ satisfying

$$
\left\{\begin{array}{l}
u_{\lambda} \in W^{1,2}(0, T ; H), u_{\lambda}(t) \in D\left(\partial_{H} \tilde{\psi}\right), \forall t \in(0, T), \\
t \mapsto \tilde{\psi}\left(u_{\lambda}(t)\right), \tilde{\varphi}_{\lambda}\left(t, u_{\lambda}(t)\right) \text { is absolutely continuous on }[0, T] .
\end{array}\right.
$$

Now, we are going to establish a couple of a priori estimates for the solution $u_{\lambda} \in W^{1,2}(0, T ; H)$ of $(\mathrm{CP})_{\lambda}(\lambda>0)$ in the following lemmas.

Lemma 3.3. There exists a constant $K_{1}$ such that

$$
\begin{aligned}
& \sup _{t \in[0, T]}\left|u_{\lambda}(t)\right|_{H} \leq K_{1}, \\
& \sup _{t \in[0, T]} \tilde{\varphi}_{\lambda}\left(t, u_{\lambda}(t)\right) \leq K_{1}, \\
& \int_{0}^{T}\left|\frac{d u_{\lambda}}{d \tau}(\tau)\right|^{2} d \tau \leq K_{1}, \\
& \sup _{t \in[0, T]}\left|u_{\lambda}(t)\right|_{X} \leq K_{1} .
\end{aligned}
$$

Proof. Multiplying $(\mathrm{CP})_{\lambda}$ by $d u_{\lambda}(t) / d t$,

$$
\begin{aligned}
& \left(\frac{d u_{\lambda}}{d t}(t), \frac{d u_{\lambda}}{d t}(t)\right)_{H}+\left(\partial_{H} \tilde{\varphi}_{\lambda}\left(t, u_{\lambda}(t)\right), \frac{d u_{\lambda}}{d t}(t)\right)_{H} \\
& -\left(\partial_{H} \tilde{\psi}\left(u_{\lambda}(t)\right), \frac{d u_{\lambda}}{d t}(t)\right)_{H} \\
= & \left(f_{\lambda}(t), \frac{d u_{\lambda}}{d t}(t)\right)_{H} .
\end{aligned}
$$

Then, by Proposition 2.2,

$$
\begin{aligned}
& \left|\frac{d u_{\lambda}}{d t}(t)\right|_{H}^{2}+\frac{d}{d t} \tilde{\varphi}_{\lambda}\left(t, u_{\lambda}(t)\right)-\frac{d}{d t} \tilde{\psi}\left(u_{\lambda}(t)\right) \\
= & \left(f_{\lambda}(t), \frac{d u_{\lambda}}{d t}(t)\right)_{H}+\frac{d}{d t} \tilde{\varphi}_{\lambda}\left(t, u_{\lambda}(t)\right)-\left(\partial_{H} \tilde{\varphi}_{\lambda}\left(t, u_{\lambda}(t)\right), \frac{d u_{\lambda}}{d t}(t)\right)_{H} .
\end{aligned}
$$

Moreover, by (A.1) and Akagi [4],

$$
\begin{aligned}
& \left|\left(\partial_{H} \tilde{\varphi}_{\lambda}\left(t, u_{\lambda}(t)\right), \frac{d u_{\lambda}}{d t}(t)\right)_{H}-\frac{d}{d t} \tilde{\varphi}_{\lambda}\left(t, u_{\lambda}(t)\right)\right| \\
\leq & |\dot{a}(t)|\left|\partial_{H} \tilde{\varphi}_{\lambda}\left(t, u_{\lambda}(t)\right)\right|_{X^{*}}\left(\tilde{\varphi}_{\lambda}\left(t, u_{\lambda}(t)\right)+1\right)^{1 / p}+|\dot{b}(t)|\left(\tilde{\varphi}_{\lambda}\left(t, u_{\lambda}(t)\right)+1\right) .
\end{aligned}
$$

Hence, with (A.3) we have

$$
\begin{aligned}
& \left|\left(\partial_{H} \tilde{\varphi}_{\lambda}\left(t, u_{\lambda}(t)\right), \frac{d u_{\lambda}}{d t}(t)\right)_{H}-\frac{d}{d t} \tilde{\varphi}_{\lambda}\left(t, u_{\lambda}(t)\right)\right| \\
\leq & \left(C_{2}^{1 / p^{\prime}}|\dot{a}(t)|+|\dot{b}(t)|\right)\left(\tilde{\varphi}_{\lambda}\left(t, u_{\lambda}(t)\right)+1\right) .
\end{aligned}
$$


Applying this to the above inequality, we get

$$
\begin{aligned}
& \left|\frac{d u_{\lambda}}{d t}(t)\right|_{H}^{2}+\frac{d}{d t} \tilde{\varphi}_{\lambda}\left(t, u_{\lambda}(t)\right)-\frac{d}{d t} \tilde{\psi}\left(u_{\lambda}(t)\right) \\
\leq & \left(f_{\lambda}(t), \frac{d u_{\lambda}}{d t}(t)\right)_{H}+\left(C_{2}^{1 / p^{\prime}}|\dot{a}(t)|+|\dot{b}(t)|\right)\left(\tilde{\varphi}_{\lambda}\left(t, u_{\lambda}(t)\right)+1\right) .
\end{aligned}
$$

Now, we integrate it over $(0, t)$ to have

$$
\begin{aligned}
& \int_{0}^{t}\left|\frac{d u_{\lambda}}{d \tau}(\tau)\right|_{H}^{2} d \tau+\tilde{\varphi}_{\lambda}\left(t, u_{\lambda}(t)\right) \\
\leq & \tilde{\varphi}_{\lambda}\left(0, u_{0}\right)+\tilde{\psi}\left(u_{\lambda}(t)\right)-\tilde{\psi}\left(u_{0}\right)+\int_{0}^{t}\left(f_{\lambda}(\tau), \frac{d u_{\lambda}}{d \tau}(\tau)\right)_{H} d \tau \\
& +\int_{0}^{t}\left(C_{2}^{1 / p^{\prime}}|\dot{a}(\tau)|+|\dot{b}(\tau)|\right)\left(\tilde{\varphi}_{\lambda}\left(\tau, u_{\lambda}(\tau)\right)+1\right) d \tau \\
\leq & \tilde{\varphi}_{\lambda}\left(0, u_{0}\right)+\tilde{\psi}\left(u_{\lambda}(t)\right)-\tilde{\psi}\left(u_{0}\right)+\frac{1}{2} \int_{0}^{t}\left|f_{\lambda}(\tau)\right|_{H}^{2} d \tau \\
& +\frac{1}{2} \int_{0}^{t}\left|\frac{d u_{\lambda}}{d \tau}(\tau)\right|_{H}^{2} d \tau+\int_{0}^{t}\left(C_{2}^{1 / p^{\prime}}|\dot{a}(\tau)|+|\dot{b}(\tau)|\right)\left(\tilde{\varphi}_{\lambda}\left(\tau, u_{\lambda}(\tau)\right)+1\right) d \tau .
\end{aligned}
$$

Then, by (A.4),

$$
\begin{aligned}
& \frac{1}{2} \int_{0}^{t}\left|\frac{d u_{\lambda}}{d \tau}(\tau)\right|_{H}^{2} d \tau+\tilde{\varphi}_{\lambda}\left(t, u_{\lambda}(t)\right) \\
\leq & \tilde{\varphi}_{\lambda}\left(0, u_{0}\right)-\tilde{\psi}\left(u_{0}\right)+\frac{1}{2} \int_{0}^{t}\left|f_{\lambda}(\tau)\right|_{H}^{2} d \tau+\int_{0}^{t}\left(C_{2}^{1 / p^{\prime}}|\dot{a}(\tau)|+|\dot{b}(\tau)|\right) d \tau \\
& +C_{3}\left(\tilde{\varphi}_{\lambda}\left(t, u_{\lambda}(t)\right)+1\right)+\int_{0}^{t}\left(C_{2}^{1 / p^{\prime}}|\dot{a}(\tau)|+|\dot{b}(\tau)|\right)\left(\tilde{\varphi}_{\lambda}(\tau, u(\tau)) d \tau .\right.
\end{aligned}
$$

At this point, using the fact $\frac{d}{d t}\left|u_{\lambda}(t)\right|_{H} \leq\left|\frac{d u_{\lambda}}{d t}(t)\right|_{H}$, we have $\mu \frac{d}{d t}\left|u_{\lambda}(t)\right|_{H}^{2} \leq$ $2 \mu^{2}\left|u_{\lambda}(t)\right|_{H}^{2}+\frac{1}{2}\left|\frac{d u_{\lambda}}{d t}(t)\right|_{H}^{2}$ for all $\mu>0$. It implies

$$
\mu\left|u_{\lambda}(t)\right|_{H}^{2}-\mu\left|u_{0}\right|_{H}^{2}-2 \mu^{2} \int_{0}^{t}\left|u_{\lambda}(\tau)\right|_{H}^{2} d \tau \leq \frac{1}{2} \int_{0}^{t}\left|\frac{d u_{\lambda}}{d \tau}(\tau)\right|_{H}^{2} d \tau
$$

Hence, by putting $\mu=1-C_{3}$ and (A.4),

$$
\begin{aligned}
& \left|u_{\lambda}(t)\right|_{H}^{2}+\tilde{\varphi}_{\lambda}\left(t, u_{\lambda}(t)\right) \\
\leq & \frac{1}{\mu}\left[\mu\left|u_{0}\right|_{H}^{2}+\tilde{\varphi}_{\lambda}\left(0, u_{0}\right)+\tilde{\psi}\left(u_{0}\right)+C_{4} T+C_{3}+\frac{1}{2} \int_{0}^{t}\left|f_{\lambda}(\tau)\right|_{H}^{2} d \tau\right]
\end{aligned}
$$


DIFFERENTIAL EQUATIONS WITH TIME-DEPENDENT SUBDIFFERENTIALS 469

$$
\begin{aligned}
& \left.+2 \mu \int_{0}^{t}\left|u_{\lambda}(\tau)\right|_{H}^{2} d \tau+C_{4} \int_{0}^{t} \tilde{\varphi}_{\lambda}(\tau, u(\tau))\right) d \tau \\
\leq & C_{5}+C_{6} \int_{0}^{t}\left(\left|u_{\lambda}(\tau)\right|_{H}^{2}+\tilde{\varphi}_{\lambda}(\tau, u(\tau))\right) d \tau,
\end{aligned}
$$

where

$$
\begin{gathered}
C_{4}=\sup _{0 \leq t \leq T}\left(C_{2}^{1 / p^{\prime}}|\dot{a}(t)|+|\dot{b}(t)|\right) \\
C_{5}=\frac{1}{\mu}\left[\mu\left|u_{0}\right|_{H}^{2}+\tilde{\varphi}_{\lambda}\left(0, u_{0}\right)+\tilde{\psi}\left(u_{0}\right)+C_{4} T+C_{3}+\frac{1}{2} \int_{0}^{t}\left|f_{\lambda}(\tau)\right|_{H}^{2} d \tau\right]
\end{gathered}
$$

and

$$
C_{6}=\max \left\{2 \mu, C_{4}\right\}
$$

since $f_{\lambda}$ is bounded in $W^{1, p^{\prime}}\left(0, T ; X^{*}\right) \cap L^{2}(0, T ; H)$. By the Gronwall's inequality,

$$
\left|u_{\lambda}(t)\right|_{H}^{2}+\tilde{\varphi}_{\lambda}\left(t, u_{\lambda}(t)\right) \leq C_{5} \exp \left(C_{6} T\right):=K_{1}
$$

for all $t \in[0, T]$. Therefore, we have the conclusions.

Lemma 3.4. There exists a constant $K_{2}$ such that

$$
\begin{aligned}
& \sup _{t \in[0, T]}\left|\partial \tilde{\varphi}_{\lambda}\left(t, u_{\lambda}(t)\right)\right|_{X^{*}}^{p^{\prime}} \leq K_{2}, \\
& \sup _{t \in[0, T]}\left|J_{\lambda}\left(t, u_{\lambda}(t)\right)\right|_{H} \leq K_{2},
\end{aligned}
$$

where $J_{\lambda}(t, \cdot)=\left(I+\lambda \partial_{H} \tilde{\varphi}(t, \cdot)\right)^{-1}$

Proof. By (A3), $\left|\partial \tilde{\varphi}_{\lambda}\left(t, u_{\lambda}(t)\right)\right|_{X^{*}}^{p^{\prime}} \leq C_{2}\left(\tilde{\varphi}_{\lambda}\left(t, u_{\lambda}(t)\right)+1\right) \leq C_{2}\left(K_{1}+1\right)$ for all $t \in[0, T]$ and for sufficiently small $\lambda>0$. Hence, $\sup _{t \in[0, T]}\left|\partial \tilde{\varphi}_{\lambda}\left(t, u_{\lambda}(t)\right)\right|_{X^{*}}^{p^{\prime}} \leq$ $K_{2}$. By Lemma 3.3,

$$
\begin{aligned}
\left|J_{\lambda}\left(t, u_{\lambda}(t)\right)\right|_{H} & \leq\left|J_{\lambda}\left(t, u_{\lambda}(t)\right)-u_{\lambda}(t)\right|_{H}+\left|u_{\lambda}(t)\right|_{H} \\
& \leq K_{1}+\lambda\left|\partial \tilde{\varphi}_{\lambda}\left(t, u_{\lambda}(t)\right)\right|_{H} \\
& \leq \lambda K_{2}+K_{1}:=K_{2} .
\end{aligned}
$$

Therefore, $\sup _{t \in[0, T]}\left|J_{\lambda}\left(t, u_{\lambda}(t)\right)\right|_{H} \leq K_{2}$.

Since the solutions $\left\{u_{\lambda}\right\}_{\lambda>0}$ are have the properties as mentioned the above lemmas, we show the existence of a function $u$ from $\left\{u_{\lambda}\right\}_{\lambda>0}$.

Lemma 3.5. There exists $u \in C_{w}([0, T] ; X) \cap W^{1,2}(0, T ; H)$ such that

$$
\begin{aligned}
& u_{\lambda_{n}} \rightarrow u \quad \text { weakly in } L^{2}(0, T ; X) \cap W^{1,2}(0, T ; H), \\
& u_{\lambda_{n}}(t) \rightarrow u(t) \quad \text { weakly in } H \quad \text { for all } t \in[0, T]
\end{aligned}
$$

as $n \rightarrow \infty$, where $\left\{\lambda_{n}\right\}>0$ is a sequence of real numbers such that $\lambda_{n} \rightarrow 0$ as $n \rightarrow \infty$. Moreover $u(t) \rightarrow u_{0}$ strongly in $H$ as $t \rightarrow 0+$. 
Proof. Since $H$ and $V$ are reflexive (2), (4) and (5) imply (8), which also yields $u \in C([0, T] ; H)$. Moreover, let $q \in[1, \infty)$ be fixed. Then by $(2)$, we can extract a subsequence $\left\{\lambda_{n}^{q}\right\}$ of $\left\{\lambda_{n}\right\}$ depending on $q$ such that $u_{\lambda_{n}^{q}}-u_{0} \rightarrow u-u_{0}$ weakly in $L^{q}(0, T ; H)$. Hence it is obvious that $u_{\lambda_{n}^{q}}-u_{0} \rightarrow u-u_{0}$ weakly in $L^{q}(0, t ; H)$ for any $t \in[0, T]$. Therefore since $u_{\lambda_{n}^{q}}(0)=u_{0}$, it follows from (4) that

$$
\begin{aligned}
\left\|u-u_{0}\right\|_{L^{q}(0, t ; H)} & \leq \liminf _{\lambda_{n}^{q} \rightarrow 0}\left\|u_{\lambda_{n}^{q}}-u_{0}\right\|_{L^{q}(0, t ; H)} \\
& \leq \liminf _{\lambda_{n}^{q} \rightarrow 0}\left(\int_{0}^{t}\left(\int_{0}^{\tau}\left|\frac{d u_{\lambda_{n}^{q}}}{d s}(s)\right|_{H}^{2}\right)^{q / 2} \tau^{q / 2} d \tau\right)^{1 / q} \\
& \leq K_{1}^{1 / 2}\left(\frac{2}{q+2}\right)^{1 / q} t^{(1 / 2+1 / q)}
\end{aligned}
$$

Thus we have

$$
\begin{aligned}
\left|u(t)-u_{0}\right|_{H} & \leq \sup _{\tau \in[0, t]}\left|u(\tau)-u_{0}\right|_{H} \\
& =\lim _{q \rightarrow+\infty}\left\|u-u_{0}\right\|_{L^{q}(0, t ; H)} \leq K_{1}^{1 / 2} t^{1 / 2}
\end{aligned}
$$

for all $t \in[0, T]$, which implies $u(t) \rightarrow u_{0}$ strongly in $H$ as $t \rightarrow+0$.

Now, let $t \in[0, T]$ be fixed. Since $u_{\lambda_{n}}(0)=u(0)=u_{0}$, we have from (5) that

$$
\left(u_{\lambda_{n}}(t)-u(t), \phi\right)_{H}=\int_{0}^{t}\left(\frac{d u_{\lambda_{n}}(\tau)}{d \tau}-\frac{d u(\tau)}{d \tau}, \phi\right)_{H} d \tau \rightarrow 0
$$

for all $\phi \in H$ and $t \in[0, T]$. It implies (9). Moreover, by (5) and (9), for any $t \in[0, T]$, we can take a subsequence $\left\{\lambda_{n}^{t}\right\}$ of $\left\{\lambda_{n}\right\}$ depending on $t$ such that

$$
u_{\lambda_{n}^{t}}(t) \rightarrow u(t) \quad \text { weakly in } X \text {. }
$$

It then follows from (5) that $|u(t)|_{X} \leq \liminf _{\lambda_{n}^{t} \rightarrow 0}\left|u_{\lambda_{n}^{t}}\right|_{X} \leq K_{1}$, where $K_{1}$ is independent of $t$. Therefore, we conclude that $u(t) \in X$ for all $t \in[0, T]$ and $\sup _{t \in[0, T]}|u(t)|_{X} \leq K_{1}<+\infty$. Hence, for all $t \in[0, T]$ and $\left\{t_{n}\right\}$ with $t_{n} \rightarrow t$ as $n \rightarrow+\infty$, there exist a subsequence $\left\{t_{n_{k}}\right\}$ of $\left\{t_{n}\right\}$ and $w \in X$ such that $u\left(t_{n_{k}}\right) \rightarrow w$ weakly in $X$ as $n_{k} \rightarrow+\infty$. On the other hand, $u\left(t_{n_{k}}\right) \rightarrow u(t)$ strongly in $H$ as $n_{k} \rightarrow+\infty$, since $u \in C_{w}([0, T] ; X)$. Then, by virtue of $X \subset H \equiv H^{*} \subset X^{*}$, we find $w=u(t)$. Whence it follows $u \in C_{w}([0, T] ; X)$.

Proof of Theorem 3.1 Since $\sup _{t \in[0, T]}\left|\partial \tilde{\varphi}_{\lambda_{n}}\left(t, u_{\lambda_{n}}(t)\right)\right|_{X^{*}}^{p^{\prime}} \leq K_{2}$ by (6), it is obvious that there exists $h \in L^{p^{\prime}}\left(0, T ; X^{*}\right)$ such that

$$
\partial \tilde{\varphi}_{\lambda_{n}}\left(\cdot, u_{\lambda_{n}}(\cdot)\right) \rightarrow h \quad \text { weakly in } \quad L^{p^{\prime}}\left(0, T ; X^{*}\right) .
$$

Since $\partial \tilde{\varphi}_{\lambda_{n}}\left(t, u_{\lambda_{n}}(t)\right) \in \partial \tilde{\varphi}\left(t, J_{\lambda_{n}}\left(t, u_{\lambda_{n}}(t)\right)\right) \subset \partial \varphi\left(t, u_{\lambda_{n}}(t)\right), h(t) \in \partial \varphi(t, u(t))$ for a.e. $t \in(0, T)$ by the demiclosedness of maximal monotone operator and Proposition 1.1 of [8]. 
Let $g_{\lambda_{n}}(t)=-f_{\lambda_{n}}(t)+\frac{d u_{\lambda_{n}}}{d t}(t)+h_{\lambda_{n}}(t) \in \partial_{H} \tilde{\psi}\left(u_{\lambda_{n}}(t)\right)$. Then, since $f_{\lambda_{n}}$ is bounded in $W^{1, p^{\prime}}\left(0, T ; X^{*}\right), \int_{0}^{T}\left|\frac{d u_{\lambda_{n}}}{d t}(t)\right|_{X^{*}}^{2} d t \leq K$ for some constant $K$ by (4) and (6). Hence, there exists $g \in L^{2}\left(0, T ; X^{*}\right)$ such that

$$
g_{\lambda_{n}} \rightarrow g \text { weakly in } L^{2}\left(0, T ; X^{*}\right),
$$

where $g(t)=-f(t)+\frac{d u}{d t}(t)+h(t)$. Moreover, from $(\mathrm{CP})_{\lambda_{n}}$ we get by integrating the product of $g_{\lambda_{n}}(t)$ and $u_{\lambda_{n}}(t)$ over $(0, T)$ that

$$
\begin{aligned}
& \int_{0}^{T}\left\langle g_{\lambda_{n}}(t), u_{\lambda_{n}}(t)\right\rangle d t \\
= & \int_{0}^{T}\left\langle-f_{\lambda_{n}}(t)+\frac{d u_{\lambda_{n}}}{d t}(t)+\partial_{H} \tilde{\varphi}_{\lambda_{n}}\left(t, u_{\lambda_{n}}(t)\right), u_{\lambda_{n}}(t)\right\rangle d t \\
= & \int_{0}^{T}\left\langle-f_{\lambda_{n}}(t), u_{\lambda_{n}}(t)\right\rangle d t+\int_{0}^{T}\left\langle\partial_{H} \tilde{\varphi}_{\lambda_{n}}\left(t, u_{\lambda_{n}}(t)\right), u_{\lambda_{n}}(t)\right\rangle d t \\
& +\frac{1}{2}\left|u_{\lambda_{n}}(T)\right|_{H}^{2}-\frac{1}{2}\left|u_{0}\right|_{H}^{2} .
\end{aligned}
$$

Since $f_{\lambda_{n}} \rightarrow f$ strongly in $W^{1, p^{\prime}}\left(0, T ; X^{*}\right)$, it follows

$$
\begin{aligned}
& \limsup _{\lambda_{n} \rightarrow 0} \int_{0}^{T}\left\langle g_{\lambda_{n}}(t), u_{\lambda_{n}}(t)\right\rangle d t \\
= & \lim _{\lambda_{n} \rightarrow 0} \int_{0}^{T}\left\langle-f_{\lambda_{n}}(t), u_{\lambda_{n}}(t)\right\rangle d t+\frac{1}{2} \limsup _{\lambda_{n} \rightarrow 0}\left|u_{\lambda_{n}}(T)\right|_{H}^{2}-\frac{1}{2}\left|u_{0}\right|_{H}^{2} \\
& +\lim _{\lambda_{n} \rightarrow 0} \int_{0}^{T}\left\langle\partial_{H} \tilde{\varphi}_{\lambda_{n}}\left(t, u_{\lambda_{n}}(t)\right), u_{\lambda_{n}}(t)\right\rangle d t \\
= & \int_{0}^{T}\left\langle-f(t)+\frac{d u(t)}{d t}+h(t), u(t)\right\rangle d t .
\end{aligned}
$$

By Lemma 1.3 of [5] and Proposition 1.1 of [8], it follows from (8) and (10) that $g(t)=-f(t)+d u(t) / d t+h(t) \in \partial \psi(u(t))$ for a.e. $t \in(0, T)$. This completes our proof.

\section{References}

[1] R. A. Adams, Sobolev Spaces, Academic Press, New York, 1975.

[2] G. Akagi, Doubly nonlinear evolution equations governed by time-dependent subdifferentials in reflexive Banach spaces, J. Differential Equations 231 (2006), no. 1, 32-56.

[3] G. Akagi and M. Otani, Evolution inclusions governed by the difference of two subdifferentials in reflexive Banach spaces, J. Differential Equations 209 (2005), no. 2, $392-415$.

[4] - Time-dependent constraint problems arising from macroscopic critical-state models for type-II superconductivity and their approximations, Adv. Math. Sci. Appl. 14 (2004), no. 2, 683-712.

[5] V. Barbu, Nonlinear Semigroups and Differential Equations in Banach Spaces, Noordhoff Intl. Publ., Leyden, 1976. 
[6] H. Brézis, Opérateurs maximaux monotones et semi-groupes de contractions dans les espaces de Hilbert, Math. Studies, Vol. 5, North-Holland, Amsterdam/New York, 1973.

[7] H. Ishii, Asymptotic stability and blowing up of solutions of some nonlinear equations, J. Differential Equations 26 (1977), no. 2, 291-319.

[8] N. Kenmochi, Some nonlinear parabolic variational inequalities, Israel J. Math. 22 (1975), no. 3-4, 304-331.

[9] Y. Koi and J. Watanabe, On nonlinear evolution equations with a difference term of subdifferentials, Proc. Japan Acad. 52 (1976), no. 8, 413-416.

[10] J. L. Lions, Quelques méthodes de résolution des problèmes aux limites non linéaires, Dunod; Gauthier-Villars, Paris 1969

[11] M. Ôtani, Nonmonotone perturbations for nonlinear parabolic equations associated with subdifferential operators, J. Differential Equations 46 (1982), no. 2, 268-299.

KIYEON SHIN

Department of Mathematics

Pusan National University

PusAn 609-735, Korea

E-mail address: kyshin@pusan.ac.kr

JINGYO JEONG

Department of Mathematics

Pusan National University

Pusan 609-735, Korea

E-mail address: jingyo11@pusan.ac.kr 